\title{
A new family of discrete Painlevé equations and associated linearisable systems
}
A. Ramani, B. Grammaticos

To cite this article: A. Ramani, B. Grammaticos (2013) A new family of discrete Painlevé equations and associated linearisable systems, Journal of Nonlinear Mathematical Physics 20: Supplement 1, 153-164, DOI:

https://doi.org/10.1080/14029251.2013.862444

To link to this article: https://doi.org/10.1080/14029251.2013.862444

Published online: 04 January 2021 


\title{
A new family of discrete Painlevé equations and associated linearisable systems
}

\author{
A. Ramani \\ Centre de Physique Théorique, Ecole Polytechnique, CNRS \\ 91128 Palaiseau, France \\ ramani@cpht.polytechnique.fr \\ B. Grammaticos \\ IMNC, Université Paris VII \& XI, CNRS, UMR 8165, Bât. 440 \\ 91406 Orsay, France \\ grammaticos@imnc.in2p3.fr \\ Received 27 February 2012 \\ Accepted 1 June 2012
}

\begin{abstract}
We derive discrete systems which result from a second, not studied up to now, form of the $q-\mathrm{P}_{\mathrm{VI}}$ equation. The derivation is based on two different procedures: "limits" and "degeneracies". We obtain several new discrete Painlevé equations along with some linearisable systems. The parallel between the results for the standard form of $q-\mathrm{P}_{\mathrm{VI}}$ and those of the new one is also established.
\end{abstract}

Keywords: Integrability; Painlevé equations; discretisation; linearisability; deautonomisation.

PACS classification: 37K10, 39A12

\section{Introduction}

With almost 20 years of intense investigations on discrete Painlevé equations [1] the claim of discovery of a new family may sound presumptuous. It is the aim of the analysis that follows to dispell any doubts and show that, despite the voluminous existing body of results, it is indeed possible to find new integrable systems of discrete Painlevé type.

It all started with our recent work [2] on linearisable QRT [3] mappings. While deriving them systematically, based on our prior classification [4] of the QRT $A_{1}$ matrices, we observed that the one associated to the $q-\mathrm{P}_{\mathrm{VI}}$ family had a different form from all the others. (We assume at this point that the reader is familiar with the theory of QRT mappings. A very brief summary thereof is given in the Appendix). Indeed while all the other canonical forms of the $A_{1}$ matrices are of lower-right triangular form, only the $A_{1}$ associated to $q-\mathrm{P}_{\mathrm{VI}}$ has the diagonal form

$$
A_{1}=\left(\begin{array}{ccc}
1 & 0 & 0 \\
0-1-z^{2} & 0 \\
0 & 0 & z^{2}
\end{array}\right)
$$

However, as we have shown there, it is possible to bring $A_{1}$ to a lower-right triangular form through a variable transformation. The price is that the resulting $q-\mathrm{P}_{\mathrm{VI}}$ has a different form. Indeed when $A_{1}$ 
is taken equal to

$$
A_{1}=\left(\begin{array}{lcr}
0 & 0 & 1 \\
0 & z+1 / z & 0 \\
1 & 0 & 0
\end{array}\right)
$$

we find that the equations of the $q-\mathrm{P}_{\mathrm{VI}}$ family assume the form

$$
\frac{\left(z x_{n+1}+x_{n}\right)\left(x_{n}+z x_{n-1}\right)}{\left(x_{n+1}+z x_{n}\right)\left(z x_{n}+x_{n-1}\right)}=F\left(x_{n}\right)
$$

while the ones related to (1) are of the form

$$
\frac{\left(x_{n+1} x_{n}-z^{2}\right)\left(x_{n} x_{n-1}-z^{2}\right)}{\left(x_{n+1} x_{n}-1\right)\left(x_{n} x_{n-1}-1\right)}=f\left(x_{n}\right)
$$

The transformation which allows to bring equations of the form (4) to equations of the form (3) consists in inverting every other $x$ and introducing the appropriate gauge. Let us illustrate this on the asymmetric $q$ - $\mathrm{P}_{\mathrm{VI}}[5]$ equation

$$
\begin{aligned}
& \frac{\left(x_{n+1} y_{n}-z^{2}\right)\left(y_{n} x_{n}-z^{2}\right)}{\left(x_{n+1} y_{n}-1\right)\left(y_{n} x_{n}-1\right)}=\frac{a y_{n}^{4}+b z y_{n}^{3}+c z^{2} y_{n}^{2}+d z^{3} y_{n}+e z^{4}}{a y_{n}^{4}+f y_{n}^{3}+g y_{n}^{2}+h y_{n}+e} \\
& \frac{\left(x_{n} y_{n}-z^{2}\right)\left(y_{n-1} x_{n}-z^{2}\right)}{\left(x_{n} y_{n}-1\right)\left(y_{n-1} x_{n}-1\right)}=\frac{e x_{n}^{4}+d z x_{n}^{3}+c z^{2} x_{n}^{2}+b z^{3} x_{n}+a z^{4}}{e x_{n}^{4}+h x_{n}^{3}+g x_{n}^{2}+f x_{n}+a}
\end{aligned}
$$

Usually (5) is presented with $a=e=1$, which is possible, without loss of generality, provided $a e \neq 0$. Now we invert $x$ and multiply by $-z$, i.e. $x \rightarrow-z / x$ while keeping $y$ as is. We find

$$
\begin{aligned}
& \frac{\left(z x_{n+1}+y_{n}\right)\left(y_{n}+z x_{n}\right)}{\left(x_{n+1}+z y_{n}\right)\left(z y_{n}+x_{n}\right)}=\frac{1}{z^{2}} \frac{a y_{n}^{4}+b z y_{n}^{3}+c z^{2} y_{n}^{2}+d z^{3} y_{n}+e z^{4}}{a y_{n}^{4}-f y_{n}^{3}+g y_{n}^{2}-h y_{n}+e} \\
& \frac{\left(z y_{n}+x_{n}\right)\left(z y_{n-1}+x_{n}\right)}{\left(y_{n}+z x_{n}\right)\left(y_{n-1}+z x_{n}\right)}=\frac{1}{z^{2}} \frac{a x_{n}^{4}+f z x_{n}^{3}+g z^{2} x_{n}^{2}+h z^{3} x_{n}+e z^{4}}{a x_{n}^{4}-b x_{n}^{3}+c x_{n}^{2}-d x_{n}+e}
\end{aligned}
$$

where we have changed the signs of $f$ and $h$ between (5) and (6) so as to have only positive signs in the numerators. A symmetric form can also be obtained for(6): it suffices to take $f=b, g=c$ and $h=d$ in order to find

$$
\frac{\left(z x_{n+1}+x_{n}\right)\left(x_{n}+z x_{n-1}\right)}{\left(x_{n+1}+z x_{n}\right)\left(z x_{n}+x_{n-1}\right)}=\frac{1}{z^{2}} \frac{a x_{n}^{4}+b z x_{n}^{3}+c z^{2} x_{n}^{2}+d z^{3} x_{n}+e z^{4}}{a x_{n}^{4}-b x_{n}^{3}+c x_{n}^{2}-d x_{n}+e}
$$

At this point one may understandably wonder whether the title of the paper is the proper one. After all, if the new family is obtained from that of $\mathrm{P}_{\mathrm{VI}}$ already presented in [6] by a straightforward transformation, the moniker "new" would be hardly justified. However this is not the case. In order to make our argument more precise let us present an explicit example (more will be given in the discussion). Suppose we take $e=0$ in (6). Going back to (5) we remark that both rhs are now ratios of cubic polynomials. However in the first equation the ratio of the highest terms is exactly unity while in the second it is not. This situation is not usually considered in the constructive approach for discrete Painlevé equations, based on the deautonomisation of QRT mappings. In particular it 
has not been considered in our study [6] of the limits and degeneracies of the $q-\mathrm{P}_{\mathrm{VI}}$ equation. The consequence of this is that, while the main equation of the new family is just $q-\mathrm{P}_{\mathrm{VI}}$ itself written in a different way, the remaining equations of the family may be new (and it turns out that some are). In what follows we are going to construct carefully the various limits and degeneracies of $q-\mathrm{P}_{\mathrm{VI}}$ in the form (6). We shall obtain their nonautonomous form, using the standard deautonomisation procedure based on the singularity confinement approach. Along the way we are going also to present systems not of the discrete Painlevé kind but which are linearisable (no such systems having been found in the form (6) of $\left.q-\mathrm{P}_{\mathrm{VI}}\right)$.

\section{The limits}

In order to present the limits of (6) we start from its nonautonomous form. As already noticed in [2] the proper desautonomisation of (6) necessitates the introduction of $z^{2}$ in lieu of $z$. We have thus

$$
\begin{aligned}
& \frac{\left(\zeta_{n} z_{n+1} x_{n+1}+y_{n}\right)\left(y_{n}+\zeta_{n} z_{n} x_{n}\right)}{\left(x_{n+1}+\zeta_{n} z_{n+1} y_{n}\right)\left(\zeta_{n} z_{n} y_{n}+x_{n}\right)}=\frac{1}{\zeta_{n}^{2}} \frac{a y_{n}^{4}+b \zeta_{n} y_{n}^{3}+c \zeta_{n}^{2} y_{n}^{2}+d \zeta_{n}^{3} y_{n}+e \zeta_{n}^{4}}{a y_{n}^{4}-f y_{n}^{3}+g y_{n}^{2}-h y_{n}+e} \\
& \frac{\left(\zeta_{n} z_{n} y_{n}+x_{n}\right)\left(z_{n} \zeta_{n-1} y_{n-1}+x_{n}\right)}{\left(y_{n}+\zeta_{n} z_{n} x_{n}\right)\left(y_{n-1}+z_{n} \zeta_{n-1} x_{n}\right)}=\frac{1}{z_{n}^{2}} \frac{a x_{n}^{4}+f z_{n} x_{n}^{3}+g z_{n}^{2} x_{n}^{2}+h z_{n}^{3} x_{n}+e z_{n}^{4}}{a x_{n}^{4}-b x_{n}^{3}+c x_{n}^{2}-d x_{n}+e}
\end{aligned}
$$

where $z_{n}=z_{0} \lambda^{n}$ and moreover we have $z_{n} z_{n+1}=\zeta_{n}^{2}$ i.e. $\zeta_{n}=z_{n+1 / 2}$. In order to proceed to the various limits it is interesting to rewrite the rhs of (8) as a product

$$
\begin{aligned}
& \frac{\left(\zeta_{n} z_{n+1} x_{n+1}+y_{n}\right)\left(y_{n}+\zeta_{n} z_{n} x_{n}\right)}{\left(x_{n+1}+\zeta_{n} z_{n+1} y_{n}\right)\left(\zeta_{n} z_{n} y_{n}+x_{n}\right)}=\frac{\left(y_{n}+\alpha \zeta_{n}\right)\left(y_{n}+\beta \zeta_{n}\right)\left(y_{n}+\gamma \zeta_{n}\right)\left(y_{n}+\delta \zeta_{n}\right)}{\left(\zeta_{n} y_{n}-\varepsilon\right)\left(\zeta_{n} y_{n}-\eta\right)\left(\zeta_{n} y_{n}-\theta\right)\left(\zeta_{n} y_{n}-\kappa\right)} \\
& \frac{\left(\zeta_{n} z_{n} y_{n}+x_{n}\right)\left(z_{n} \zeta_{n-1} y_{n-1}+x_{n}\right)}{\left(y_{n}+\zeta_{n} z_{n} x_{n}\right)\left(y_{n-1}+z_{n} \zeta_{n-1} x_{n}\right)}=\frac{\left(x_{n}+z_{n} \varepsilon\right)\left(x_{n}+z_{n} \eta\right)\left(x_{n}+z_{n} \theta\right)\left(x_{n}+z_{n} \kappa\right)}{\left(z_{n} x_{n}-\alpha\right)\left(z_{n} x_{n}-\beta\right)\left(z_{n} x_{n}-\gamma\right)\left(z_{n} x_{n}-\delta\right)}
\end{aligned}
$$

where the $\alpha, \beta, \ldots, \kappa$ are defined in terms of the $a, b, \ldots, h$ and are satisfy the constraint

$$
\alpha \beta \gamma \delta=\varepsilon \eta \theta \kappa
$$

Thus the number of effective parameters is just 6 , since an overall scaling of $x$ and $y$ is allowed. A symmetric form of the mapping can also be obtained if we take $\varepsilon=\alpha, \eta=\beta, \theta=\gamma$ and $\kappa=\delta$. We obtain thus

$$
\frac{\left(z_{n} z_{n+1} x_{n+1}+x_{n}\right)\left(x_{n}+z_{n-1} z_{n} x_{n-1}\right)}{\left(x_{n+1}+z_{n} z_{n+1} x_{n}\right)\left(z_{n-1} z_{n} x_{n}+x_{n-1}\right)}=\frac{\left(x_{n}+\alpha z_{n}\right)\left(x_{n}+\beta z_{n}\right)\left(x_{n}+\gamma z_{n}\right)\left(x_{n}+\delta z_{n}\right)}{\left(z_{n} x_{n}-\alpha\right)\left(z_{n} x_{n}-\beta\right)\left(z_{n} x_{n}-\gamma\right)\left(z_{n} x_{n}-\delta\right)}
$$

Here we have just three parameters (a scaling of $x$ being always possible). 
The limits of (9) and (11) can be obtained by taking the parameters to 0 or to $\infty$. Let us start from (9) and take $\alpha \rightarrow 0, \varepsilon \rightarrow 0$. We find

$$
\begin{gathered}
\frac{\left(\zeta_{n} z_{n+1} x_{n+1}+y_{n}\right)\left(y_{n}+\zeta_{n} z_{n} x_{n}\right)}{\left(x_{n+1}+\zeta_{n} z_{n+1} y_{n}\right)\left(\zeta_{n} z_{n} y_{n}+x_{n}\right)}=\frac{1}{\zeta_{n}} \frac{\left(y_{n}+\beta \zeta_{n}\right)\left(y_{n}+\gamma \zeta_{n}\right)\left(y_{n}+\delta \zeta_{n}\right)}{\left(\zeta_{n} y_{n}-\eta\right)\left(\zeta_{n} y_{n}-\theta\right)\left(\zeta_{n} y_{n}-\kappa\right)} \\
\frac{\left(\zeta_{n} z_{n} y_{n}+x_{n}\right)\left(z_{n} \zeta_{n-1} y_{n-1}+x_{n}\right)}{\left(y_{n}+\zeta_{n} z_{n} x_{n}\right)\left(y_{n-1}+z_{n} \zeta_{n-1} x_{n}\right)}=\frac{1}{z_{n}} \frac{\left(x_{n}+z_{n} \eta\right)\left(x_{n}+z_{n} \theta\right)\left(x_{n}+z_{n} \kappa\right)}{\left(z_{n} x_{n}-\beta\right)\left(z_{n} x_{n}-\gamma\right)\left(z_{n} x_{n}-\delta\right)}
\end{gathered}
$$

where the remaining parameters are not constrained anymore. An analogous expression holds in the symmetric case. Next we take $\alpha \rightarrow \infty, \varepsilon \rightarrow \infty$. We find

$$
\begin{gathered}
\frac{\left(\zeta_{n} z_{n+1} x_{n+1}+y_{n}\right)\left(y_{n}+\zeta_{n} z_{n} x_{n}\right)}{\left(x_{n+1}+\zeta_{n} z_{n+1} y_{n}\right)\left(\zeta_{n} z_{n} y_{n}+x_{n}\right)}=-\frac{\eta \theta \kappa \zeta_{n}}{\beta \gamma \delta} \frac{\left(y_{n}+\beta \zeta_{n}\right)\left(y_{n}+\gamma \zeta_{n}\right)\left(y_{n}+\delta \zeta_{n}\right)}{\left(\zeta_{n} y_{n}-\eta\right)\left(\zeta_{n} y_{n}-\theta\right)\left(\zeta_{n} y_{n}-\kappa\right)} \\
\frac{\left(\zeta_{n} z_{n} y_{n}+x_{n}\right)\left(z_{n} \zeta_{n-1} y_{n-1}+x_{n}\right)}{\left(y_{n}+\zeta_{n} z_{n} x_{n}\right)\left(y_{n-1}+z_{n} \zeta_{n-1} x_{n}\right)}=-\frac{\beta \gamma \delta z_{n}}{\eta \theta \kappa} \frac{\left(x_{n}+z_{n} \eta\right)\left(x_{n}+z_{n} \theta\right)\left(x_{n}+z_{n} \kappa\right)}{\left(z_{n} x_{n}-\beta\right)\left(z_{n} x_{n}-\gamma\right)\left(z_{n} x_{n}-\delta\right)}
\end{gathered}
$$

where, again, the 6 remaining parameters are not constrained anymore. Clearly, in the symmetric case, the prefactor is simply $-z_{n}$. When two pairs of parameters, say $\alpha, \varepsilon$ and $\beta, \eta$ go to zero we obtain simply

$$
\begin{gathered}
\frac{\left(\zeta_{n} z_{n+1} x_{n+1}+y_{n}\right)\left(y_{n}+\zeta_{n} z_{n} x_{n}\right)}{\left(x_{n+1}+\zeta_{n} z_{n+1} y_{n}\right)\left(\zeta_{n} z_{n} y_{n}+x_{n}\right)}=\frac{1}{\zeta_{n}^{2}} \frac{\left(y_{n}+\gamma \zeta_{n}\right)\left(y_{n}+\delta \zeta_{n}\right)}{\left(\zeta_{n} y_{n}-\theta\right)\left(\zeta_{n} y_{n}-\kappa\right)} \\
\frac{\left(\zeta_{n} z_{n} y_{n}+x_{n}\right)\left(z_{n} \zeta_{n-1} y_{n-1}+x_{n}\right)}{\left(y_{n}+\zeta_{n} z_{n} x_{n}\right)\left(y_{n-1}+z_{n} \zeta_{n-1} x_{n}\right)}=\frac{1}{z_{n}^{2}} \frac{\left(x_{n}+z_{n} \theta\right)\left(x_{n}+z_{n} \kappa\right)}{\left(z_{n} x_{n}-\gamma\right)\left(z_{n} x_{n}-\delta\right)}
\end{gathered}
$$

Combining the case $\alpha \rightarrow \infty, \varepsilon \rightarrow \infty$ with $\beta \rightarrow 0, \eta \rightarrow 0$ we find

$$
\begin{aligned}
& \frac{\left(\zeta_{n} z_{n+1} x_{n+1}+y_{n}\right)\left(y_{n}+\zeta_{n} z_{n} x_{n}\right)}{\left(x_{n+1}+\zeta_{n} z_{n+1} y_{n}\right)\left(\zeta_{n} z_{n} y_{n}+x_{n}\right)}=\rho \frac{\left(y_{n}+\gamma \zeta_{n}\right)\left(y_{n}+\delta \zeta_{n}\right)}{\left(\zeta_{n} y_{n}-\theta\right)\left(\zeta_{n} y_{n}-\kappa\right)} \\
& \frac{\left(\zeta_{n} z_{n} y_{n}+x_{n}\right)\left(z_{n} \zeta_{n-1} y_{n-1}+x_{n}\right)}{\left(y_{n}+\zeta_{n} z_{n} x_{n}\right)\left(y_{n-1}+z_{n} \zeta_{n-1} x_{n}\right)}=\frac{1}{\rho} \frac{\left(x_{n}+z_{n} \theta\right)\left(x_{n}+z_{n} \kappa\right)}{\left(z_{n} x_{n}-\gamma\right)\left(z_{n} x_{n}-\delta\right)}
\end{aligned}
$$

where $\rho$ is a free parameter. Finally we consider the case where two pairs of parameters go to $\infty$ and find

$$
\begin{gathered}
\frac{\left(\zeta_{n} z_{n+1} x_{n+1}+y_{n}\right)\left(y_{n}+\zeta_{n} z_{n} x_{n}\right)}{\left(x_{n+1}+\zeta_{n} z_{n+1} y_{n}\right)\left(\zeta_{n} z_{n} y_{n}+x_{n}\right)}=\frac{\theta \kappa \zeta_{n}^{2}}{\gamma \delta} \frac{\left(y_{n}+\gamma \zeta_{n}\right)\left(y_{n}+\delta \zeta_{n}\right)}{\left(\zeta_{n} y_{n}-\theta\right)\left(\zeta_{n} y_{n}-\kappa\right)} \\
\frac{\left(\zeta_{n} z_{n} y_{n}+x_{n}\right)\left(z_{n} \zeta_{n-1} y_{n-1}+x_{n}\right)}{\left(y_{n}+\zeta_{n} z_{n} x_{n}\right)\left(y_{n-1}+z_{n} \zeta_{n-1} x_{n}\right)}=\frac{\gamma \delta z_{n}^{2}}{\theta \kappa} \frac{\left(x_{n}+z_{n} \theta\right)\left(x_{n}+z_{n} \kappa\right)}{\left(z_{n} x_{n}-\gamma\right)\left(z_{n} x_{n}-\delta\right)}
\end{gathered}
$$

In the symmetric limit of (15) the prefactor is just $\rho=-1$, while for (16) we find simply $z_{n}^{2}$. The last cases to study are the limits when three pairs of parameters take special values. In the case of 
three pairs of zeros we find

$$
\begin{aligned}
& \frac{\left(\zeta_{n} z_{n+1} x_{n+1}+y_{n}\right)\left(y_{n}+\zeta_{n} z_{n} x_{n}\right)}{\left(x_{n+1}+\zeta_{n} z_{n+1} y_{n}\right)\left(\zeta_{n} z_{n} y_{n}+x_{n}\right)}=\frac{1}{\zeta_{n}^{3}}\left(\frac{y_{n}+\delta \zeta_{n}}{\zeta_{n} y_{n}-\kappa}\right) \\
& \frac{\left(\zeta_{n} z_{n} y_{n}+x_{n}\right)\left(z_{n} \zeta_{n-1} y_{n-1}+x_{n}\right)}{\left(y_{n}+\zeta_{n} z_{n} x_{n}\right)\left(y_{n-1}+z_{n} \zeta_{n-1} x_{n}\right)}=\frac{1}{z_{n}^{3}}\left(\frac{x_{n}+z_{n} \kappa}{z_{n} x_{n}-\delta}\right)
\end{aligned}
$$

When two pairs of zeros are combined with one pair of infinities we obtain the equation

$$
\begin{aligned}
& \frac{\left(\zeta_{n} z_{n+1} x_{n+1}+y_{n}\right)\left(y_{n}+\zeta_{n} z_{n} x_{n}\right)}{\left(x_{n+1}+\zeta_{n} z_{n+1} y_{n}\right)\left(\zeta_{n} z_{n} y_{n}+x_{n}\right)}=\frac{\rho}{\zeta_{n}}\left(\frac{y_{n}+\delta \zeta_{n}}{\zeta_{n} y_{n}-\kappa}\right) \\
& \frac{\left(\zeta_{n} z_{n} y_{n}+x_{n}\right)\left(z_{n} \zeta_{n-1} y_{n-1}+x_{n}\right)}{\left(y_{n}+\zeta_{n} z_{n} x_{n}\right)\left(y_{n-1}+z_{n} \zeta_{n-1} x_{n}\right)}=\frac{1}{\rho z_{n}}\left(\frac{x_{n}+z_{n} \kappa}{z_{n} x_{n}-\delta}\right)
\end{aligned}
$$

where $\rho$ is a free parameter. Similarly when two pairs of infinities are combined with one pair of zeros we obtain the equation

$$
\begin{gathered}
\frac{\left(\zeta_{n} z_{n+1} x_{n+1}+y_{n}\right)\left(y_{n}+\zeta_{n} z_{n} x_{n}\right)}{\left(x_{n+1}+\zeta_{n} z_{n+1} y_{n}\right)\left(\zeta_{n} z_{n} y_{n}+x_{n}\right)}=\rho \zeta_{n}\left(\frac{y_{n}+\delta \zeta_{n}}{\zeta_{n} y_{n}-\kappa}\right) \\
\frac{\left(\zeta_{n} z_{n} y_{n}+x_{n}\right)\left(z_{n} \zeta_{n-1} y_{n-1}+x_{n}\right)}{\left(y_{n}+\zeta_{n} z_{n} x_{n}\right)\left(y_{n-1}+z_{n} \zeta_{n-1} x_{n}\right)}=\frac{z_{n}}{\rho}\left(\frac{x_{n}+z_{n} \kappa}{z_{n} x_{n}-\delta}\right)
\end{gathered}
$$

where $\rho$ is again a free parameter. Finally we have the case where three pairs of parameters go to infinity. In this case we find the equation

$$
\begin{aligned}
& \frac{\left(\zeta_{n} z_{n+1} x_{n+1}+y_{n}\right)\left(y_{n}+\zeta_{n} z_{n} x_{n}\right)}{\left(x_{n+1}+\zeta_{n} z_{n+1} y_{n}\right)\left(\zeta_{n} z_{n} y_{n}+x_{n}\right)}=-\frac{\kappa \zeta_{n}^{3}}{\delta}\left(\frac{y_{n}+\delta \zeta_{n}}{\zeta_{n} y_{n}-\kappa}\right) \\
& \frac{\left(\zeta_{n} z_{n} y_{n}+x_{n}\right)\left(z_{n} \zeta_{n-1} y_{n-1}+x_{n}\right)}{\left(y_{n}+\zeta_{n} z_{n} x_{n}\right)\left(y_{n-1}+z_{n} \zeta_{n-1} x_{n}\right)}=-\frac{\delta z_{n}^{3}}{\kappa}\left(\frac{x_{n}+z_{n} \kappa}{z_{n} x_{n}-\delta}\right)
\end{aligned}
$$

In the symmetric limit of (18) we find that the prefactor is $\rho=-1$ while for (19) we obtain $\rho=1$.

While there appears to exist a profusion of different limits of (9) some simple relations between them allow, in a sense, to reduce the number of cases on has to consider. We remark that (11) is invariant under the transformation $x \rightarrow 1 / x$ (and, obviously, $y \rightarrow 1 / y$ in the asymmetric case), $z \rightarrow 1 / z$ provided we invert also all the parameters. In that case the limit where a pair of parameters goes to 0 is dual to the case where a pair goes to $\infty$. In that sense (12) is dual to (13), (14) dual to (16), (17) dual to (20), (18) dual to (19) while (15) is a self-dual equation. Equation (12) appears to be new, at least to the authors' knowledge, while the case of (15) will be examined in the discussion. On the other hand (14), (17) and (18) were identified [2], in their autonomous forms, as being linearisable. While the first and last belong to the variety of what we have dubbed linearisable systems of the third kind, (17) is a mapping of the Gambier type. In all of the above systems the variable $z_{n}$ is of the form $z_{n}=z_{0} \lambda^{n}$ as in the initial equation. However for the Gambier-type 
mapping (17) we have shown in [7] that it can be written in a form involving a free function of the independent variable. We found thus, in the symmetric case the equation

$$
\left(\frac{z_{n} z_{n+1} x_{n+1}+x_{n}}{x_{n+1}+z_{n} z_{n+1} x_{n}}\right)\left(\frac{x_{n}+z_{n} z_{n-1} x_{n-1}}{z_{n} z_{n-1} x_{n}+x_{n-1}}\right)=\frac{1}{z_{n+1} z_{n} z_{n-1}}\left(\frac{x_{n}+z_{n}}{z_{n} x_{n}-1}\right)
$$

where $z_{n}$ is now a free function of $n$. (An analogous expression holds for the asymmetric case).

\section{The degeneracies}

The term "degeneracy" was introduced in [8] in order to designate equations obtained from the initial one under the assumption that some condition holds allowing to introduce a simplification from numerator and denominator of one or more factors. In this case the integrability conditions are not the same as for the initial system. They must be obtained afresh and in general lead to dependence on the independent variable not present in the initial system. In what follows we shall obtain the integrability constraints for the degeneracies of (9) by appling the singularity confinement [9] criterion.

The first case is when one factorisation occurs allowing us to write the system under consideration as

$$
\begin{aligned}
& \frac{\left(\zeta_{n} z_{n+1} x_{n+1}+y_{n}\right)\left(y_{n}+\zeta_{n} z_{n} x_{n}\right)}{\left(x_{n+1}+\zeta_{n} z_{n+1} y_{n}\right)\left(\zeta_{n} z_{n} y_{n}+x_{n}\right)}=\frac{\left(y_{n}-\phi_{n}\right)\left(y_{n}+\gamma \zeta_{n}\right)\left(y_{n}+\delta \zeta_{n}\right)}{\left(\psi_{n} y_{n}-\rho_{n}\right)\left(\zeta_{n} y_{n}-\theta\right)\left(\zeta_{n} y_{n}-\kappa\right)} \\
& \frac{\left(\zeta_{n} z_{n} y_{n}+x_{n}\right)\left(z_{n} \zeta_{n-1} y_{n-1}+x_{n}\right)}{\left(y_{n}+\zeta_{n} z_{n} x_{n}\right)\left(y_{n-1}+z_{n} \zeta_{n-1} x_{n}\right)}=\frac{\left(x_{n}-\chi_{n}\right)\left(x_{n}+z_{n} \theta\right)\left(x_{n}+z_{n} \kappa\right)}{\left(\omega_{n} x_{n}-\sigma_{n}\right)\left(z_{n} x_{n}-\gamma\right)\left(z_{n} x_{n}-\delta\right)}
\end{aligned}
$$

where $\phi_{n}=\chi_{n+1 / 2}, \psi_{n}=\omega_{n+1 / 2}$ and $\rho_{n}=\sigma_{n+1 / 2}$. First we remark that using a gauge on $z_{n}, \zeta_{n}$ we may put $\gamma \delta=\theta \kappa$ and using a scaling on $x_{n}, y_{n}$ we may still reduce the number of these four parameters to just two. However, keeping an asymmetric form, in view of the higher periodicities usually obtained, does not present any advantage. Thus we introduce a symmetric form of (22) and work with it in what follows. We rewrite (22) (redefining the various parameters lest we run out of greek letters) as

$$
\left(\frac{z_{n} z_{n+1} x_{n+1}+x_{n}}{x_{n+1}+z_{n} z_{n+1} x_{n}}\right)\left(\frac{x_{n}+z_{n} z_{n-1} x_{n-1}}{z_{n} z_{n-1} x_{n}+x_{n-1}}\right)=\frac{\left(x_{n}-\phi_{n}\right)\left(x_{n}+\omega_{n} z_{n}\right)\left(x_{n}+\chi_{n} z_{n}\right)}{\left(\psi_{n} x_{n}-\rho_{n}\right)\left(z_{n} x_{n}-\tau_{n}\right)\left(z_{n} x_{n}-\sigma_{n}\right)}
$$

where we have $\omega_{n} \chi_{n}=\tau_{n} \sigma_{n}$. The use of the singularity confinement criterion leads to the following dependence of the various parameters on $n$. We find $\log z_{n}=a n+b+c j^{n}+d j^{2 n}$ where $j^{3}=1$. We also find that $\rho_{n}$ is a parity dependent constant i.e. $\log \rho_{n}=h+k(-1)^{n}$, and $\psi_{n}=z_{n-1} z_{n+1}$, $\phi_{n}=\rho_{n} z_{n-1} z_{n+1}$. Moreover we have $\log \chi_{n}=e+g(-1)^{n}, \log \sigma_{n}=e-g(-1)^{n}, \log \omega_{n}=f-g(-1)^{n}$, $\log \tau_{n}=f+g(-1)^{n}$, corresponding to the alternating constants $(\gamma, \theta)$ and $(\delta, \kappa)$. By a scaling of $x$ we can set $f=-e$. Thus the total number of degrees of freedom is 7 and we expect the geometry of the transformations of(23) to be described [10] by the affine Weyl group $E_{7}^{(1)}$. Once the form of (23) is established we can consider its various limits. Taking the limit $\omega \rightarrow 0$ and $\tau \rightarrow 0$, or $\omega \rightarrow \infty$ and $\tau \rightarrow \infty$, leads to the disappearance of a parameter from the equation and thus we expect its geometry 
to be described by the affine Weyl group $E_{6}^{(1)}$. The case $\omega \rightarrow 0, \tau \rightarrow 0$, for instance, has the form

$$
\left(\frac{z_{n} z_{n+1} x_{n+1}+x_{n}}{x_{n+1}+z_{n} z_{n+1} x_{n}}\right)\left(\frac{x_{n}+z_{n} z_{n-1} x_{n-1}}{z_{n} z_{n-1} x_{n}+x_{n-1}}\right)=\frac{1}{z_{n}} \frac{\left(x_{n}-\rho_{n} z_{n-1} z_{n+1}\right)\left(x_{n}+\chi_{n} z_{n}\right)}{\left(z_{n-1} z_{n+1} x_{n}-\rho_{n}\right)\left(z_{n} x_{n}-\sigma_{n}\right)}
$$

where $z_{n}, \rho_{n}, \chi_{n}, \sigma_{n}$ are given just above. We remark that (24) could also have been obtained from(12) by a degeneracy procedure involving the first two terms. Taking both pairs $(\omega, \tau)$ and $(\chi, \sigma)$ to 0 or to $\infty$ leads to a linearisable mapping. Taking $\chi \rightarrow 0, \sigma \rightarrow 0$ in (24) we obtain

$$
\left(\frac{z_{n} z_{n+1} x_{n+1}+x_{n}}{x_{n+1}+z_{n} z_{n+1} x_{n}}\right)\left(\frac{x_{n}+z_{n} z_{n-1} x_{n-1}}{z_{n} z_{n-1} x_{n}+x_{n-1}}\right)=\frac{1}{z_{n}^{2}}\left(\frac{x_{n}-\rho_{n} z_{n-1} z_{n+1}}{z_{n-1} z_{n+1} x_{n}-\rho_{n}}\right)
$$

However when one pair, say $(\omega, \tau)$ is taken to 0 while the other $(\chi, \sigma)$ is taken to $\infty$ we obtain a discrete Painlevé equation involving 5 degrees of freedom the geometry of which is expected to be described by the affine Weyl group $D_{5}^{(1)}$

$$
\left(\frac{z_{n} z_{n+1} x_{n+1}+x_{n}}{x_{n+1}+z_{n} z_{n+1} x_{n}}\right)\left(\frac{x_{n}+z_{n} z_{n-1} x_{n-1}}{z_{n} z_{n-1} x_{n}+x_{n-1}}\right)=-\frac{\chi_{n}}{\sigma_{n}}\left(\frac{x_{n}-\rho_{n} z_{n-1} z_{n+1}}{z_{n-1} z_{n+1} x_{n}-\rho_{n}}\right)
$$

where the prefactor is just $-\exp \left(2 g(-1)^{n}\right)$. This mapping could also have been obtained from (15) by a degeneracy procedure. Finally taking the limit $\rho \rightarrow 0$, respectively $\rho \rightarrow \infty$, we find a linearisable mapping of the form(14), respectively (16), where now $z_{n}$ has a ternary freedom.

The next case we are going to examine is one where two factorisations occur. This can be done in two different ways. We first assume that the common factor exists between the last two factors of the rhs of (23). In this case we can as well work with the symmetric form of the mapping. With a little bit of hindsight we introduce the form

$$
\left(\frac{z_{n} z_{n+1} x_{n+1}+x_{n}}{x_{n+1}+z_{n} z_{n+1} x_{n}}\right)\left(\frac{x_{n}+z_{n} z_{n-1} x_{n-1}}{z_{n} z_{n-1} x_{n}+x_{n-1}}\right)=\frac{1}{z_{n+1} z_{n}^{2} z_{n-1}} \frac{\left(x_{n}-\phi_{n}\right)\left(x_{n}-\chi_{n}\right)}{\left(x_{n}-\rho_{n}\right)\left(x_{n}-\sigma_{n}\right)}
$$

where $\phi \chi=z_{n+1}^{2} z_{n}^{4} z_{n-1}^{2} \rho \sigma$. We remark that, the way the equation is written, only the quantity $z_{n+1} z_{n}$ (and its downshift) has a meaning. Applying the singularity confinement criterion we find thus that $\log z_{n+1} z_{n}=a(2 n+1)+2 b+c(-1)^{n}$ and $\log \phi_{n}=2 a n+2 b+d(-1)^{n}+e i^{n}+f(-i)^{n}+g, \log \chi_{n}=$ $2 a n+2 b+h(-1)^{n}-e i^{n}-f(-i)^{n}+k, \log \rho_{n}=-2 a n-2 b+d(-1)^{n}-e i^{n}-f(-i)^{n}+g, \log \sigma_{n}=$ $-2 a n-2 b+h(-1)^{n}+e i^{n}+f(-i)^{n}+k$. Moreover, using the overall scaling of $x$ we can put $k=-g$. The total number of degrees of freedom is 7 and thus the geometry of the transformations of this discrete Painlevé equation should be described by the affine Weyl group $E_{7}^{(1)}$. Having established the form of (27) we can now proceed to derive its limits. Since the pairs $(\phi, \rho)$ and $(\chi, \sigma)$ play the same role it suffices to consider the limits of one of those, say $(\chi, \sigma)$. Taking $\chi \rightarrow 0$ and $\sigma \rightarrow 0$ (respectively $\chi \rightarrow \infty$ and $\sigma \rightarrow \infty$ ) we obtain a linearisable equation, similar to (25) but where $z_{n}$ has a different $n$-dependence. We should point out here that, in order to proceed to these limits, we must take $k \rightarrow-\infty$ (respectively $k \rightarrow+\infty$ ). Thus the scaling of $x$ can be introduced only after the limit, allowing, for instance, to put $g=0$.

The second case of double degeneracy corresponds to appearance of a common factor in the first two terms of the rhs of (22). The fully asymmetric equation has the form

$$
\frac{\left(\zeta_{n} z_{n+1} x_{n+1}+y_{n}\right)\left(y_{n}+\zeta_{n} z_{n} x_{n}\right)}{\left(x_{n+1}+\zeta_{n} z_{n+1} y_{n}\right)\left(\zeta_{n} z_{n} y_{n}+x_{n}\right)}=\frac{\left(y_{n}-\phi_{n}\right)\left(y_{n}+\delta \zeta_{n}\right)}{\left(\psi_{n} y_{n}-\rho_{n}\right)\left(\zeta_{n} y_{n}-\kappa\right)}
$$




$$
\frac{\left(\zeta_{n} z_{n} y_{n}+x_{n}\right)\left(z_{n} \zeta_{n-1} y_{n-1}+x_{n}\right)}{\left(y_{n}+\zeta_{n} z_{n} x_{n}\right)\left(y_{n-1}+z_{n} \zeta_{n-1} x_{n}\right)}=\frac{\left(x_{n}-\chi_{n}\right)\left(x_{n}+z_{n} \kappa\right)}{\left(\omega_{n} x_{n}-\sigma_{n}\right)\left(z_{n} x_{n}-\delta\right)}
$$

where, as in the case of(22), $\phi_{n}=\chi_{n+1 / 2}, \psi_{n}=\omega_{n+1 / 2}$ and $\rho_{n}=\sigma_{n+1 / 2}$. However, as for(24), it is more convenient to work with the symmetric form, which, again with hindsight, we write as

$$
\left(\frac{z_{n} z_{n+1} x_{n+1}+x_{n}}{x_{n+1}+z_{n} z_{n+1} x_{n}}\right)\left(\frac{x_{n}+z_{n} z_{n-1} x_{n-1}}{z_{n} z_{n-1} x_{n}+x_{n-1}}\right)=\frac{\left(x_{n}+\phi_{n} z_{n+1} z_{n} z_{n-1}\right)\left(x_{n}+\chi_{n} z_{n}\right)}{\left(z_{n+1} z_{n} z_{n-1} x_{n}-\rho_{n}\right)\left(z_{n} x_{n}-\sigma_{n}\right)}
$$

Applying the singularity confinement criterion, and using, as an auxiliary for the final verification, the criterion of algebraic entropy [11] we obtain the following: $\log z_{n}=a n+b+c j^{n}+d j^{2 n}$, (with $\left.j^{3}=1\right), \log \chi_{n}=f+g(-1)^{n}, \log \sigma_{n}=f-g(-1)^{n}, \log \phi_{n}=h+k j^{n}+l j^{2 n}-g(-1)^{n}$ and $\log \rho_{n}=$ $h+k j^{n}+l j^{2 n}+g(-1)^{n}$. Moreover, using the overall scaling of $x$ we can put $h=-f$. Just as in the case of (24) the total number of degrees of freedom is 7 . Thus the geometry of the transformations of this discrete Painlevé equation should be described by the affine Weyl group $E_{7}^{(1)}$. Two limits are possible here. Taking $\phi \rightarrow 0$ and $\rho \rightarrow 0$ (respectively $\phi \rightarrow \infty$ and $\rho \rightarrow \infty$ ) leads to a linearisable equation of the Gambier type, (21). On the other hand taking $\chi \rightarrow 0$ and $\sigma \rightarrow 0$ (respectively $\chi \rightarrow \infty$ and $\sigma \rightarrow \infty$ ) leads to a mapping with 6 degrees of freedom the geometry of which, we expect, should be described by the affine Weyl group $E_{6}^{(1)}$.

$$
\left(\frac{z_{n} z_{n+1} x_{n+1}+x_{n}}{x_{n+1}+z_{n} z_{n+1} x_{n}}\right)\left(\frac{x_{n}+z_{n} z_{n-1} x_{n-1}}{z_{n} z_{n-1} x_{n}+x_{n-1}}\right)=\frac{1}{z_{n}}\left(\frac{x_{n}+\phi_{n} z_{n+1} z_{n} z_{n-1}}{z_{n+1} z_{n} z_{n-1} x_{n}-\rho_{n}}\right)
$$

This mapping could also have been obtained from (24) by a degeneracy procedure. Here again the limit is obtained by putting $f \rightarrow-\infty$ or $f \rightarrow+\infty$ and thus the scaling of $x$ can only be performed after the limit (allowing to put $h=0$ ).

The final degeneracy leads to a rhs which is just homographic in $x$. In this case we have, again with a dose of hindsight,

$$
\left(\frac{z_{n} z_{n+1} x_{n+1}+x_{n}}{x_{n+1}+z_{n} z_{n+1} x_{n}}\right)\left(\frac{x_{n}+z_{n} z_{n-1} x_{n-1}}{z_{n} z_{n-1} x_{n}+x_{n-1}}\right)=\frac{x_{n}-\rho_{n} z_{n+1} z_{n}^{2} z_{n-1}}{z_{n+1} z_{n}^{2} z_{n-1} x_{n}-\rho_{n}}
$$

We remark again that only the quantity $z_{n+1} z_{n}$ (and its downshift) has a meaning. Applying the singularity confinement criterion we find that $\log z_{n+1} z_{n}=a n+b+c(-1)^{n}+d j^{n}+e j^{2 n}$, where $j^{3}=1$, and $\log \rho_{n}=f+g(-1)^{n}+h i^{n}+k(-i)^{n}$. Using the scaling of $x$ we can take $f=0$ and thus (31) has precisely 7 degrees of freedom. As a consequence we expect the geometry of the transformations of(31) to be described by the affine Weyl group $E_{7}^{(1)}$.

\section{Discussion}

In this paper we have set out to derive new forms for discrete Painlevé equations (and linearisable systems) related to the form (6) of $q$ - $\mathrm{P}_{\mathrm{VI}}$. The rationale behind our approach is that the $\mathrm{P}_{\mathrm{VI}}$ family, as studied in [6] under the form(5), does not encompass all possible forms of $q$-discrete Painlevé equations associated to the $E_{7}^{(1)}$ affine Weyl group (and their degenerations). In order to make this argument (already presented in the introduction) more explicit, some examples and a clarification of terminology are necessary at this point. 
In [8] the discrete Painlevé equation

$$
x_{n+1} x_{n-1}=\frac{a q_{n} x_{n}+q_{n}^{2}}{x_{n}\left(x_{n}-1\right)}
$$

with $q_{n}=q_{0} \lambda^{n}$, was identified as a limit of the $q-\mathrm{P}_{\mathrm{VI}}$ equation. Equation (32) is cast in what, in the QRT [3] terminology, is a symmetric form. An extension of (32) to an (again in the QRT terminology) asymmetric form was obtained in [12]. It has the form

$$
\begin{gathered}
x_{n+1} x_{n}=\frac{b z_{n} y_{n}+z_{n} z_{n+1}}{y_{n}\left(y_{n}-1\right)} \\
y_{n} y_{n-1}=\frac{a z_{n} x_{n}+z_{n}^{2}}{x_{n}\left(x_{n}-1\right)}
\end{gathered}
$$

with $z_{n}=q_{0} \lambda^{n / 2}$. An inspection of (33) shows that both the lhs and the rhs of the two halves have a similar stucture, their difference lying only in the appearance of different coefficients. We shall call this situation a weakly asymmetric one: equation (33) can be brought to symmetric form by taking $x_{n} \rightarrow x_{2 n}, y_{n} \rightarrow x_{2 n+1}$ and $b=a \sqrt{\lambda}$.

The opposite situation is that of equations obtained by deautonomising mappings corresponding to an asymmetric $A_{1}$ QRT matrix. They are systems where already the lhs of the two halves of the system have different forms. An example of such a discrete Painlevé equation is the system derived in [13]

$$
\begin{gathered}
x_{n+1} x_{n}=\frac{y_{n}-z_{n}}{y_{n}^{2}-a^{2}} \\
y_{n}+y_{n-1}=\frac{1}{x_{n}}+\frac{z_{n}+b}{1-x_{n}}
\end{gathered}
$$

In this case we are in the presence of a by construction strongly asymmetric equation.

However the weak and the "by construction strong" asymmetries do not exhaust all possibilities. While studying the discrete analogues of the various $\mathrm{P}_{\mathrm{III}}$ equations [14] we obtained the following form for the one-parameter $q-\mathrm{P}_{\mathrm{III}}$

$$
\begin{aligned}
& x_{n+1} x_{n}=\frac{1+q_{n} y_{n}}{y_{n}\left(y_{n}-1\right)} \\
& y_{n} y_{n-1}=\frac{a q_{n} x_{n}+1}{x_{n}^{2}}
\end{aligned}
$$

Equation (35) can be obtained from (33) with the appropriate scaling of the variable $x$ and a limiting procedure. (The parameters $a, b$ must also be scaled while $q$ must be inverted). Once the limit is taken, and(35) is obtained, there is no way to reduce the latter to a symmetric form. While this is not an asymmetry as strong as that of (34), since the lhs of (35) have similar forms, we are not in presence of a weak asymmetry. For lack of a better terminology we describe this asymmetry as a strong one.

How does all this apply to the case at hand? Already in the introduction we have hinted at the fact that some asymmetric situations were not considered in our study of limits and degeneracies 
of $q$ - $\mathrm{P}_{\mathrm{VI}}$. They are typically cases which belong to the strong asymmetric class. Having derived the limits and degeneracies of the second form of the $q-\mathrm{P}_{\mathrm{VI}}$ family it is now interesting to compare the results to those obtained in [6] and establish the possible parallels. Let us start from equation (9) and take the limit $\alpha \rightarrow 0$ and $\varepsilon \rightarrow 0$ i.e. equation (22). Next we introduce the change of variables $x_{n}=-z_{n} / X_{n}, y_{n}=Y_{n} / \zeta_{n}$. We obtain thus the system

$$
\begin{gathered}
\frac{\left(X_{n+1} Y_{n}-\zeta_{n}^{2} z_{n+1}^{2}\right)\left(X_{n} Y_{n}-\zeta_{n}^{2} z_{n}^{2}\right)}{\left(X_{n+1} Y_{n}-1\right)\left(X_{n} Y_{n}-1\right)}=\frac{\left(Y_{n}+\beta \zeta_{n}^{2}\right)\left(Y_{n}+\gamma \zeta_{n}^{2}\right)\left(Y_{n}+\delta \zeta_{n}^{2}\right)}{\left(Y_{n}-\eta\right)\left(Y_{n}-\theta\right)\left(Y_{n}-\kappa\right)} \\
\frac{\left(X_{n} Y_{n}-\zeta_{n}^{2} z_{n}^{2}\right)\left(X_{n} Y_{n-1}-z_{n}^{2} \zeta_{n-1}^{2}\right)}{\left(X_{n} Y_{n}-1\right)\left(X_{n} Y_{n-1}-1\right)}=-z_{n}^{2} \frac{\beta \gamma \delta}{\eta \theta \kappa} \frac{\left(X_{n}+z_{n}^{2} / \beta\right)\left(X_{n}+z_{n}^{2} / \gamma\right)\left(X_{n}+z_{n}^{2} \delta\right)}{\left(X_{n}-1 / \eta\right)\left(X_{n}-1 / \theta\right)\left(X_{n}-1 / \kappa\right)}
\end{gathered}
$$

We remark that the two left-hand sides of (36) have different prefactors. This is a strong asymmetry and thus (36) is absent from our classification presented in [6]. The same holds true for all the other cases of section 2 with the exception of equation (15). The latter is indeed present in [6], where it was identified as equation (2.3). Next we turn to the equations obtained in section 3. Performing the same transformation as for (36) we can establish the equivalence between the present results and those of [6]. We find thus that (23), (26), (27) and (31) correspond to the equations (2.10), (2.20), (2.16) and (2.29) of [6], while for (24) and (30) no analogue appears (since it is of strongly asymmetric form). The case of equation(29) is special. This equation should have had an analogue form among the results of [6]. For unfathomable reasons this case does not appear there. We remedy this omission by presenting its form here. By applying the singularity confinement criterion we obtain the equation

$$
\frac{\left(x_{n+1} x_{n}-z_{n+1}^{2} z_{n}^{2}\right)\left(x_{n} x_{n-1}-z_{n}^{2} z_{n-1}^{2}\right)}{\left(x_{n+1} x_{n}-1\right)\left(x_{n} x_{n-1}-1\right)}=z_{n+1} z_{n-1} \frac{\left(x_{n}-z_{n+1} z_{n}^{2} z_{n-1} \phi_{n}\right)\left(x_{n}-\chi_{n} z_{n}^{2}\right)}{\left(z_{n+1} z_{n-1} x_{n}-\rho_{n}\right)\left(x_{n}-\sigma_{n}\right)}
$$

where $\log \sigma_{n}=f(-1)^{n}, \log \chi_{n}=g(-1)^{n}, \log z_{n}=a n+b+c j^{n}+d^{2 n}$ (with $\left.j^{3}=1\right), \log \rho_{n}=$ $h(-1)^{n}+k(-j)^{n}+l\left(-j^{2}\right)^{n}$ and $\log \phi_{n}=(h+g-f)(-1)^{n}+k(-j)^{n}+l\left(-j^{2}\right)^{n}$. A degree of freedom of the form $e(-1)^{n}$ does also exist in $z_{n}$ but it disappears in the lhs of (37) and can be absorbed into the remaining coefficients in the rhs. Thus we can simply neglect this term. Moreover, since the lhs of the equation involves only the product of two consecutive $x_{n}$, an overall parity-dependent scaling can be introduced allowing, for instance to take $h=f$.

Reciprocally there exist cases, identified in our study on $q-\mathrm{P}_{\mathrm{VI}}$ which were not obtained in the present paper. In particular the limit of $q-\mathrm{P}_{\mathrm{VI}}$ obtained by putting $a=b=d=e=0$ in (5) leads to a an equation, of the form studied here, which is

$$
\begin{gathered}
\frac{\left(z x_{n+1}+y_{n}\right)\left(y_{n}+z x_{n}\right)}{\left(x_{n+1}+z y_{n}\right)\left(z y_{n}+x_{n}\right)}=\frac{c y_{n}}{f y_{n}^{2}+g y_{n}+h} \\
\frac{\left(z y_{n}+x_{n}\right)\left(z y_{n-1}+x_{n}\right)}{\left(y_{n}+z x_{n}\right)\left(y_{n-1}+z x_{n}\right)}=\frac{-f x_{n}^{2}+g z x_{n}-h z^{2}}{c z x_{n}}
\end{gathered}
$$

The asymmetry of the latter is clearly a strong one and thus it was not captured in our analysis. In a similar way all equations obtained by degeneracy of $q-\mathrm{P}_{\mathrm{VI}}$ in [6], with the exception of the ones mentioned in the previous paragraph, are not present in the present paper, corresponding to strongly asymmetric systems (but of the type of (36) where a coefficient has a fixed value of 1 , rather than that of (38) where some coefficients are 0 ). 
The analysis we just presented raises an important question concerning the derivation of discrete Painlevé equations. The usual procedure is to start from a QRT mapping and deautonomise it using an integrability criterion (singularity confinement, algebraic entropy or a combination of the two). However if one starts from an asymmetric QRT mapping one is tacitly looking only for systems which are weakly asymmetric, eschewing thus possible strong cases. The results of the present paper show that systems of the latter type abound. In some cases, like that of (38), one can obtain a strongly asymmetric result starting from an asymmetric QRT with just the proviso that certain coefficients be allowed to take a special value every other time. However there exist cases where the initial system cannot be of asymmetric QRT type but rather a QRT-type mapping with coefficients of periodicity higher than two, like the ones we introduced in [15]. This open a new field of investigation for the construction of discrete Painlevé equations (and associated linearisable mappings) which we intend to explore in some future work of ours.

\section{Appendix A. A refresher on QRT mappings}

In order to contruct the QRT mappings one starts by introducing two $3 \times 3$ matrices, $A_{0}$ and $A_{1}$, of the form

$$
A_{i}=\left(\begin{array}{ccc}
\alpha_{i} & \beta_{i} & \gamma_{i} \\
\delta_{i} & \varepsilon_{i} & \zeta_{i} \\
\kappa_{i} & \lambda_{i} & \mu_{i}
\end{array}\right)
$$

Next, one introduces the vector $\vec{X}=\left(x^{2}, x, 1\right)$ from which one constructs the vectors $\vec{F} \equiv\left(f_{1}, f_{2}, f_{3}\right)$ and $\vec{G} \equiv\left(g_{1}, g_{2}, g_{3}\right)$ through $\vec{F}=\left(\vec{X} \widetilde{A}_{0}\right) \times\left(\vec{X} \widetilde{A}_{1}\right)$ and $\vec{G}=\left(\vec{X} A_{0}\right) \times\left(\vec{X} A_{1}\right)$, where the tilde denotes the transpose of the matrix. The QRT mapping is then given by:

$$
\begin{gathered}
x_{n+1}=\frac{f_{1}\left(y_{n}\right)-x_{n} f_{2}\left(y_{n}\right)}{f_{2}\left(y_{n}\right)-x_{n} f_{3}\left(y_{n}\right)} \\
y_{n+1}=\frac{g_{1}\left(x_{n+1}\right)-y_{n} g_{2}\left(x_{n+1}\right)}{g_{2}\left(x_{n+1}\right)-y_{n} g_{3}\left(x_{n+1}\right)}
\end{gathered}
$$

This is the form of what is called the asymmetric QRT mapping. It possesses an invariant

$$
K=\frac{\alpha_{0} x^{2} y^{2}+\beta_{0} x^{2} y+\gamma_{0} x^{2}+\delta_{0} x y^{2}+\varepsilon_{0} x y+\zeta_{0} x+\kappa_{0} y^{2}+\lambda_{0} y+\mu_{0}}{\alpha_{1} x^{2} y^{2}+\beta_{1} x^{2} y+\gamma_{1} x^{2}+\delta_{1} x y^{2}+\varepsilon_{1} x y+\zeta_{1} x+\kappa_{1} y^{2}+\lambda_{1} y+\mu_{1}}
$$

When both $A_{0}$ and $A_{1}$ matrices are symmetric the mapping is called symmetric. In this case we have $g_{i}=f_{i}$ and (A.2) reduces to a single equation

$$
x_{m+1}=\frac{f_{1}\left(x_{m}\right)-x_{m-1} f_{2}\left(x_{m}\right)}{f_{2}\left(x_{m}\right)-x_{m-1} f_{3}\left(x_{m}\right)}
$$

with the identification $x_{n} \rightarrow x_{2 n}, y_{n} \rightarrow x_{2 n+1}$.

In [4] we have classified the canonical forms of the $A_{1}$ QRT matrices. Nine such forms were obtained, eight of which correspond to a symmetric $A_{1}$ while in the ninth case the $A_{1}$ is asymmetric and leads to mappings of the form (34) presented in section 4. 


\section{References}

[1] A. Ramani, B. Grammaticos, J. Hietarinta, Phys. Rev. Lett. 67 (1991) 1829.

[2] A. Ramani, B. Grammaticos, J. Satsuma, N. Mimura, J. Phys. A 44 (2011) 425201.

[3] G.R.W. Quispel, J.A.G. Roberts and C.J. Thompson, Physica D 34 (1989) 183.

[4] A. Ramani, S. Carstea, B. Grammaticos and Y. Ohta, Physica A 305 (2002) 437.

[5] B. Grammaticos, A. Ramani, Phys. Lett. A 257 (1999) 288.

[6] A. Ramani, R. Willox, B. Grammaticos, A.S. Carstea and J. Satsuma, Physica A 347 (2005) 1.

[7] B. Grammaticos, A. Ramani, J. Satsuma, R. Willox, J. Math. Phys. 53 (2012) 023506.

[8] A. Ramani, B. Grammaticos, Physica A 228 (1996), 160.

[9] B. Grammaticos, A. Ramani and V. Papageorgiou, Phys. Rev. Lett. 67 (1991), 1825.

[10] H. Sakai, Commun. Math. Phys. 220 (2001) 165.

[11] J. Hietarinta and C. M. Viallet, Phys. Rev. Lett. 81 (1998), 325.

[12] M.D. Kruskal, K.M. Tamizhmani, B. Grammaticos, A. Ramani, Reg. Chaot. Dyn. 5 (2000) 273.

[13] B. Grammaticos, Y. Ohta, A. Ramani, H. Sakai, J. Phys. A 31 (1998) 3545.

[14] A. Ramani, B. Grammaticos, T. Tamizhmani, K.M. Tamizhmani, J. Phys. A 33 (2000) 579.

[15] A. Ramani, B. Grammaticos, R. Willox, Nonlinearity 24 (2011) 113. 